\title{
Alginate-Microfibers Produced by Self-Assembly in Cell Culture Medium
}

\author{
Jeong-Hui Park, ${ }^{\dagger,+}$ Ueon Sang Shin, ${ }^{\dagger, *, *}$ and Hae-Won Kim ${ }^{\dagger, \ldots, s, *}$ \\ †Department of Nanobiomedical Science \& WCU Research Center, Dankook University Graduate School, Korea \\ "E-mail: usshin12@dankook.ac.kr \\ ${ }^{\ddagger}$ Institute of Tissue Regeneration Engineering (ITREN), Dankook University, Korea \\ ${ }^{\S}$ Department of Biomaterials Science, School of Dentistry, Dankook University, Korea. ${ }^{*}$ E-mail: kimhw@dku.edu \\ Received August 25, 2010, Accepted November 23, 2010
}

\begin{abstract}
Alginate microfibers were fabricated by self assembly of alginate monomers exuded from alginate beads $(\sim 2 \mathrm{~mm}$ in diameter) containing calcium phosphate. Upon incubation of the beads in cell culture medium at $37^{\circ} \mathrm{C}$ for a few days, fibers with a diameter of about $7 \mu \mathrm{m}$ started to sprout from the bead surface, and these grew up to about $10 \mathrm{~mm}$ in length, resulting in the beads being covered with fiber forests similar to chestnut bur. The combined system of the alginatebased microfiber forest and bead is considered to be useful as a novel 3-dimensional scaffold for cell culture and tisssue growth.
\end{abstract}

Key Words: Alginate fibers, Alginate beads, Calcium phosphate, Scaffolds

\section{Introduction}

The development of scaffolds to modulate 3-dimensional (3D) cell behaviors including adhesion, proliferation, migration and differentiation is a key aspect of tissue engineering approach. ${ }^{1}$ A range of polymeric materials, which are degradable in human body, have been engineered with different forms such as porous foams, fibrous scaffolds, and particulates, to find proper usage as $3 \mathrm{D}$ cell and tissue scaffolds. ${ }^{2}$ Alginate is a naturally derived polysaccharide that has many attractive properties including low cytotoxicity and biodegradability. Indeed, polysaccharide chains can be cross-linked by molecular self-assembly via ionic or hydrogen bonds to form hydrogels with 3D network structures that hold a large amount of water. ${ }^{3,4}$ Accordingly, alginate hydrogels have been extensively used for many biomedical purposes, including cell culturing and drug delivery. ${ }^{5-7}$ Various forms of alginate such as porous matrix, microsphere and fiber have also been developed for tissue repair. ${ }^{8,9}$ In particular, alginate fibers have been produced generally by spinning methods, such as melt-spinning, wet-spinning and electro-spinning. ${ }^{10-12}$

Herein, we report a novel generation of alginate fiber, illustrating a phenomenon where the alginate fibers were exuded from alginate beads containing calcium phosphate within an environment of culture medium. ${ }^{13-14}$ The generated alginate fibrous forest upon the sphere bead is considered to be a useful 3D matrix for in situ culturing of tissue cells and further tissue engineering.

\section{Experimental Section}

Reagents and Analyses. Sodium alginate with a high content of guluronic acid (molar ratio of mannuronic acid to guluronic acid of 0.65) and a molecular weight of 70,000 Da were purchased from Sigma (St. Louis, MO). $\alpha$-Tricalcium phosphate $\left(\alpha\right.$-TCP, $\mathrm{Ca}_{3}\left(\mathrm{PO}_{4}\right)_{2},<5 \mu \mathrm{m}$ in diameter $)$ was obtained after thermal treatment of $\mathrm{CaCO}_{3}$ with dicalcium phosphate anhydrous at $1400{ }^{\circ} \mathrm{C}$ followed by rapid cooling to room temperature. Hydroxyapatite $\left(\mathrm{HA}, \mathrm{Ca}_{10}\left(\mathrm{PO}_{4}\right)_{6}(\mathrm{OH})_{2}\right)$ powder was purchased from Alfa Aesar. Alpha modified eagle's medium (Alpha MEM, cat. No LM 008-53), fetal bovine serum (FBS), and antibiotic penicillin/streptomycin were purchased from WelGENE. Dulbecco's phosphate-buffered saline (DPBS, cat. No Cat. LB 00102) was purchased from WelGENE. Scanning electron microscopy (SEM) was conducted using a JSM7000F (JEOL, Tokyo, Japan) microscope.

Production of Alg-CaP Beads and Microfibers. An aqueous solution containing $2 \%(\mathrm{w} / \mathrm{v})$ sodium alginate and $10 \%(\mathrm{w} / \mathrm{v})$ calcium phosphate $(\mathrm{CaP}=98 \% \alpha$-TCP and $2 \% \mathrm{HA})$ was dropped through a syringe needle $(0.4 \mathrm{~mm}$ in internal diameter $)$ into $10 \mathrm{mM}$ calcium chloride solution. The calcium chloride solution was then stirred for $30 \mathrm{~min}$, which resulted in the beads being externally gelled in a spherical form. The resultant beads were then washed with PBS to remove the excess $\mathrm{CaCl}_{2}$. The asprepared Alg-CaP beads were then placed in a cell culture medium (DMEM supplemented with 10\% FBS and 1\% penicillin/ streptomycin) and incubated at $37^{\circ} \mathrm{C}$ for several days to induce the formation of alginate microfibers over the beads.

\section{Results and Discussion}

Figure 1 schematically shows the mechanism of the alginate microfiber formation from the Alg-CaP beads. When the Alg$\mathrm{CaP}$ drop is in contact with the $\mathrm{CaCl}_{2}$ solution, the surface of the bead becomes hardened, forming a thin skin gel layer. The beads were taken out and then incubated in a cell culture medium. During incubation of the Alg-CaP beads in the culture medium at $37{ }^{\circ} \mathrm{C}$ for about $5 \mathrm{~h}$, fibers began to sprout out the surface. These fibers eventually grew up to about $10 \mathrm{~mm}$ in length and about $7 \mu \mathrm{m}$ in diameter, resulting in the bead surface being completely covered with microfibrous forest after a few days.

The generation of the alginate fiber was monitored through the incubation periods up to 5 days (Figure 2a). The 3D mor- 

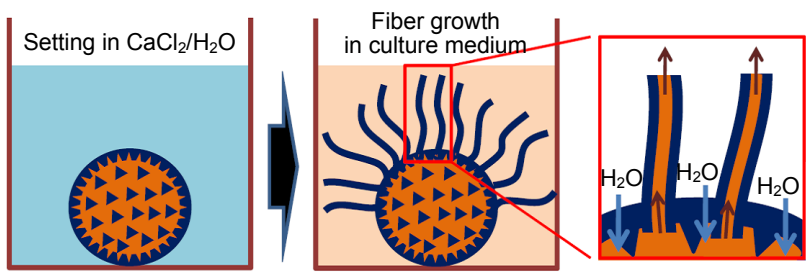

Alginate hydrogel crust $=$; Alginate solution

$\mathrm{CaP}<$

Figure 1. Schematic demonstration of the formation of alginate microfibers from the Alg-CaP bead.

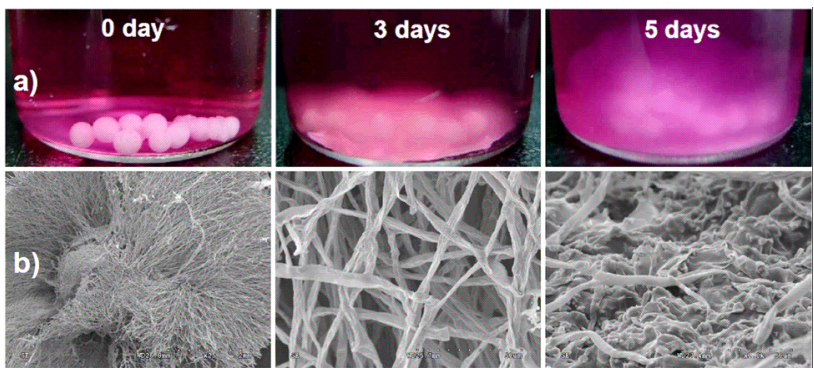

Figure 2. a) Photos of the alginate fibers formed from $\mathrm{Alg}-\mathrm{CaP}$ beads containing $10 \% \mathrm{CaP}$ after different incubation times $(0,3$ and 5 days) and b) SEM images of the alginate fibers.

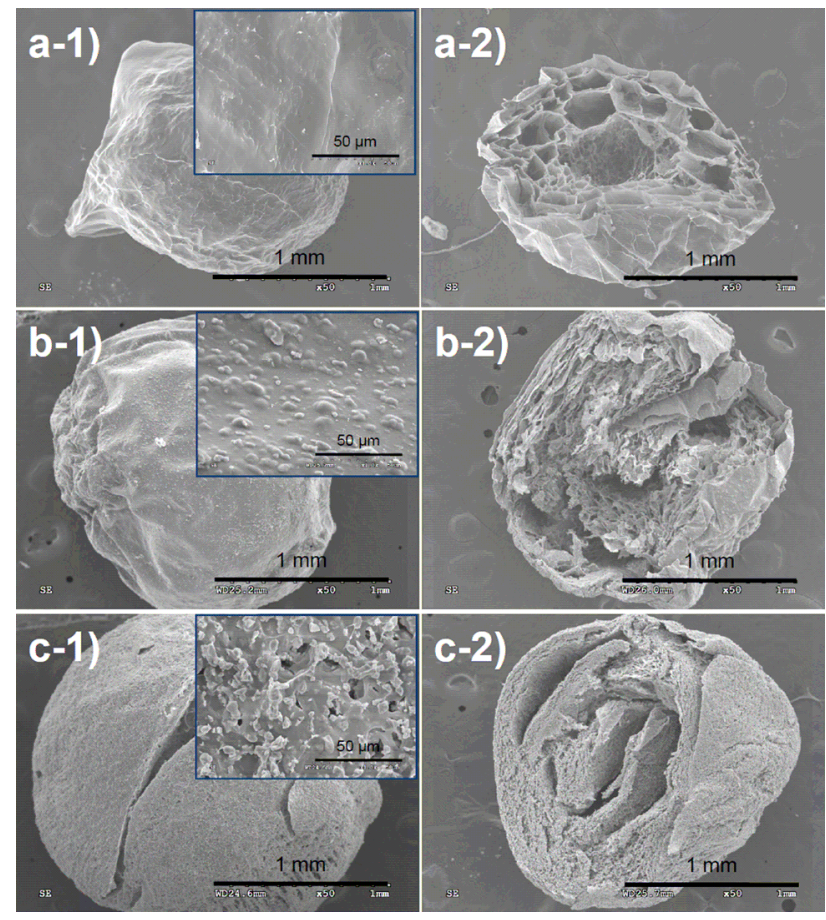

Figure 3. SEM images of beads, the surface morphologies and their cross sections after freeze drying: a) Alg bead, b) Alg-CaP bead containing $1 \%(\mathrm{w} / \mathrm{v}) \mathrm{CaP}$ and c) Alg-CaP bead containing $10 \%(\mathrm{w} / \mathrm{v}) \mathrm{CaP}$.

phologies of the fibers and the beads were examined by SEM after freeze drying the samples (Figure 2). At the fifth day, a large number of fibers upon a bead were well observed which is similar to a chestnut bur. The microfibers had a tubular structure with a thickness of about $7 \mu \mathrm{m}$. It appeared that the fibers originated from some voids or cracks which are present on the

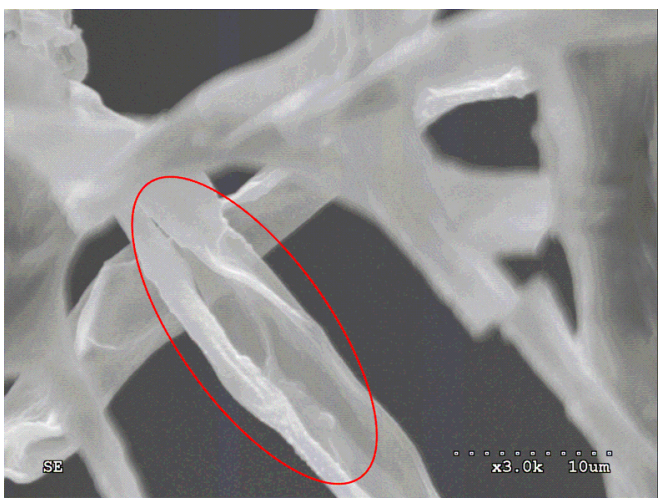

Figure 4. SEM image of the tubular fibers.

bead surface. The fully grown fibers were shown to degrade after about 10 days.

To examine how the hydrogel fibers grew from the Alg-CaP beads, the as-prepared beads with and without $\mathrm{CaP}$, Alg-CaP and Alg beads, were freeze-dried and then subjected to SEM to observe the surface and inside morphologies (Figure 3). Normally, calcium cross-linked alginate has hydrogel characteristic where the alginate monomers were 3-dimensionally networked by ionic bonds with calcium ions, containing a large amount of water. After freeze-drying the highly porous structure of inner part illustrates the hydrogel characteristic of the Alg bead (Figure 3a). However, the surface was highly dense, which was attributed to the cross-linking within $\mathrm{CaCl}_{2}$ solution.

When small amount of $\mathrm{CaP}(1 \%)$ was added, the inner porous and outer dense morphology was similar to that observed in pure alginate bead (Figure $3 \mathrm{~b}$ ). Interestingly however when the amount of $\mathrm{CaP}$ increased to $10 \%$, many voids/micropores were noticed on the surface, being in sharp contrast to the case of pure alginate bead (Figure 3c). The pore sizes were shown to be a few to ten micrometers. Moreover, the inner pore sizes appeared to be reduced a lot: from sparse macropores in pure alginate to numerous micropores in Alg-CaP containing 10\% (w/v) CaP. This microporous inner structure should be created through the interactions of alginate molecules with the $\mathrm{CaP}$ added.

Next, we addressed the mechanism by which the hydrogel fibers sprouted and grew from the bead surface during incubation in a cell culture medium. The alginate solution present at the internal part of the beads, which is loosely cross-linked, should initially come out through the voids of the bead surface. The exuding alginate should be in contact with the surrounding medium (cell culture medium, which containing $1.82 \mathrm{mM}$ of $\mathrm{Ca}$ as well), with its contact surface being hardened, and at the same time, the continual release of alginate and further surface hardening results in tubular fibrous morphology, as shown in Figure 4. Although there may be other factors to address fully the mechanism suggested herein, the formation of the alginate fibrous forest on the bead in this specified system, i. e., alginate containing $\mathrm{CaP}$ bead incubated in the $\mathrm{Ca}$-containing medium. Moreover, the fibrous beads can be potentially useful for in situ culturing of cells, as they will provide a 3D fibrous matrix for cells to migrate and populate. The biocompatibility assessment using tissue cells is currently underway. 


\section{Conclusion}

A novel alginate microfiber system produced on a bead was reported. When the alginate- $10 \% \mathrm{CaP}$ composite beads which was hardened within a $\mathrm{CaCl}_{2}$ were incubated in a cell culture medium, alginate began to sprout out to form a long tubular structure with diameter of $\sim 7 \mu \mathrm{m}$ and a length of $\sim 10 \mathrm{~mm}$. The resultant fiber forest on a bead, with morphological similarity to a chestnut bur, may be useful as an in situ cell culturing scaffold for tissue regeneration area.

Acknowledgments. This work was supported by the Priority Research Centers Program (grant\#: 2009-0093829) and WCU (World Class University) program (grant\#: R31-10069) through the National Research Foundation (NRF) funded by the Ministry of Education, Science and Technology.

\section{References}

1. Lee, K. Y.; Peters, M. C.; Anderson, K. W.; Mooney, D. J. Nature
2000, 408, 998.

2. Jang, J. H.; Castano, O.; Kim, H. W. Adv. Drug Deliv. Rev. 2009, $61,1065$.

3. Smidsrød, O.; Skjak-Braek, G. Trends Biotechnol. 1990, 8, 71.

4. Orive, G.; Tam, S. K.; Pedraz, J. L.; Halle, J. P. Biomaterials 2006, 27, 3691.

5. Eiselt, P.; Yeh, J.; Latvala, R. K.; Shea, L. D.; Mooney, D. J. Biomaterials 2000, 21, 1921.

6. Amsden, B.; Turner, N. Biotechnol. Bioeng. 1999, 65, 605.

7. Khotimchenko, Y. S.; Kovalev, V. V.; Savchenko, O. V.; Ziganshina, O. A. Marine Pharmacol. 2001, 27, 53.

8. Qin ,Y. Polym. Int. 2008, 57, 171.

9. Qin, Y. Textile Res. J. 2005, 75,165.

10. Grant, G. T.; Morris, E. R.; Rees, D. A.; Smith, P. J. C.; Thom, D. FEBS Lett. 1973, 32,195.

11. Fridrikh, S. V.; Yu, J. H.; Brenner, M. P.; Rutledge, G. C. Phys. Rev. Lett. 2003, 90, 144502

12. Bashur, C. A.; Dahlgren, L. A.; Goldstein, A. S. Biomaterials 2006, 27, 5681

13. Luginbuehl, V.; Wenk, E.; Koch, A.; Gander, B.; Merkle, H. P.; Meinel, L. Pharm. Res. 2005, 22, 940.

14. Tampieri, A.; Sandri, M.; Landi, E.; Celotti, G.; Roveri, N.; Mattioli-Belmonte, M.; Virgili, L.; Gabbanelli, F.; Biagini, G. Acta Biomater. 2005, 1, 343. 